\title{
Parametric Eyeball Model for Interactive Simulation of Ophthalmologic Surgery
}

\author{
Yiyu Cai*1 ${ }^{1}$, Chee-Kong Chui ${ }^{2}$, Yaoping Wang ${ }^{2}$, Zhenlan Wang ${ }^{2}$, \\ James H. Anderson ${ }^{3}$ \\ ${ }^{1}$ School of MPE, Nanyang Technological University, Singapore 639798 \\ ${ }^{2}$ Biomedical Lab, Kent Ridge Digital Labs, Singapore 119613 \\ ${ }^{3}$ Johns Hopkins University School of Medicine, Maryland 21205, USA \\ Email: myycai@ntu.edu.sq
}

\begin{abstract}
In this paper, we describe a parametric eyeball modeling system for real-time simulation of eye surgery. A knowledge-based approach is used to parametrically model the ophthalmologic structures. Together with the parametric eye modeling and the integration of patient-specific disease images, the ophthalmologic knowledge databases of eye diseases, eye material properties, and eye surgical instruments and procedures can help to provide better maneuverability and more realistic scenarios for eye surgical simulation. A customized Finite Element Method (FEM) is developed to analyze the deformation of the eyeball and the interactions between the bio-structures and the instruments in the surgery simulation. A phantom haptic and force feedback virtual environment is used for real-time interactive ophthalmologic surgical simulation.
\end{abstract}

\section{Introduction}

Eye diseases are among the most common and rapidly growing public health concerns. Eye surgery involves very dedicated procedures, and new techniques and device are being constantly introduced to the ophthalmology community [1-2]. The complications of ophthalmologic diseases, however, often pose difficulties for surgeons to make both timely and accurate critical decisions. Subsequently, very intensive training is required for ophthalmologic professionals in order to provide high quality standard surgical services.

Computer eye surgical simulation has been developed to provide better training for the ophthalmologic students or junior eye surgeons. Hanna et al reported their simulation work of arcuate keratomomy for astigmatism [3-4]. Sagar et al built an eye surgical simulator within a virtual reality environment [5-6]. Bio-mechanical studies for eye anatomy and pathology play a vital role in eye surgical simulation [7-10].

In this work, we aim to develop a more realistic eye surgical simulator. Patientspecific eye diseases will be considered with the simulation. This information is further enhanced by the knowledge database providing typical information and scenario in eye diseases, diseased eye material properties and eye surgery instruments and procedures. The work focuses on the Asian populations. A parametric eyeball

W. Niessen and M. Viergever (Eds.): MICCAI 2001, LNCS 2208, pp. 465-472, 2001.

(C) Springer-Verlag Berlin Heidelberg 2001 
model including the cornea, lens, sclera, retina, and choroid, etc, are developed with the capability of $3 \mathrm{D}$ variational change of the ophthalmology. A customized computational engine using FEM method is provides real-time analysis for deformation and interaction in eye surgery. The graphical simulation is seamlessly integrated with the FEM based physical modeling on the advanced OpenGL architecture and virtual haptic environment.

\section{Parametric Eyeball Modeling Using Ophthalmologic Knowledge Guidance}

\subsection{Ophthalmologic Knowledge Base}

Our online survey on eye disease was conducted in Singapore recently that shows about $80 \%$ of the 168 voting people have myopia (Figure 1). There is also a trend in the average age of the onset of pupils diagnosed with eye diseases. Eye surgery is becoming more common and popular. Traditionally, eye surgery trainees practice using animal eyeballs. There approach presnts problems. First, animals don't suffer from the same disease as humans. Next, animal eyeballs are not reusable and if a mistake is made, the eyeball must be discarded and a new one used. Also, there are differences in terms of ophthalmologic anatomy and pathology between different races, sex, age, etc.

We are building an ophthalmologic knowledge database for Asian races. The data is obtained from published literature, public domain eye disease data, and interviews including online survey. Typical data include (1) Asian eye diseases; (2) Asian eye biomechanical material property; and (3) Eye surgical instrument and procedures. The knowledge database will capture various eye diseases for the Asian population (Figure 2). The eye disease database captures information of geometry and pathology. The eye material property database keeps biomechanical values such as Young's and Shear's modules for various parts of the bio-structure. The eye instrument and procedure database include typical surgical devices and procedures used for a particular operation. With such an ophthalmologic knowledge database, simulation of different eye disease scenario becomes feasible.

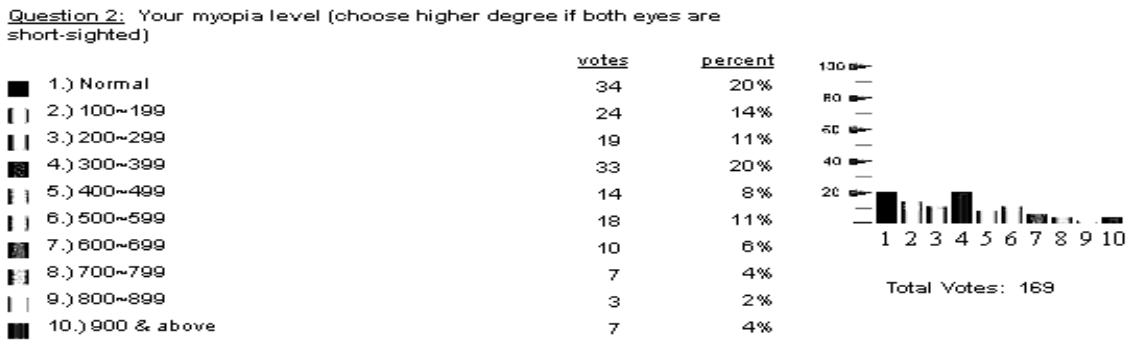

Figure 1. Online survey on eye disease in Singapore 


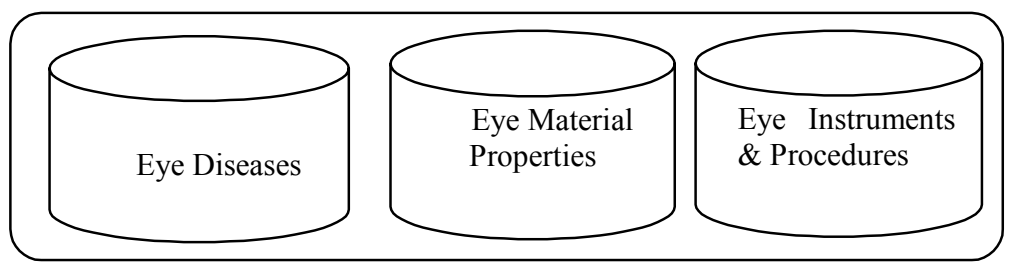

Figure 2. Ophthalmologic Knowledge Database

\subsection{Parametric Eyeball Modeling}

We model the eyeball parametrically. This allows variational change of eyeball geometry and anatomy. The cornea is transparent, and resembles a little watchglass [7-8]. In the anterior aspect, the cornea is transversely ellipsoid, whereas its posterior aspect is circular. Looked from in front, the cornea is elliptical. From the behind, the cornea appears circular. This difference is due to the fact that the sclera and conjunctiva overlap the cornea anteriorly more above and below than laterally. Ideally, the cornea forms part of the surface of a sphere. In diseased condition such as astigmatism, it is more curved in the vertical than in the horizontal meridian. The sclera forms the posterior opaque approximately five-sixths of the fibrous tunic of the eye. Its anterior portion is visible, and constitutes the "white" of the eye. The sclera is thickest behind and gradually becomes thinner when traced forwards. The choroid is firmly attached to the margin of the optic nerve, and slightly placed at the points where vessels and nerves enter it. The lens of the eye is a transparent biconvex body of crystalline appearance placed between the iris and the vitreous. Its axial diameter varies markedly with accommodation. Like all lenses, that of the eye presents two surfaces: anterior and posterior, and a border where these surfaces meet, known as the equator. The anterior surface is the segment of a sphere.

Figure 3 illustrates the eyeball model represented in a polygonal mode. Individual parts of the eyeball are parametrically represented and variation of the geometry is possible. With the ophthalmologic knowledge database, the parametric modeled eyeballs can provide high fidelity platforms for eye surgical simulation.

\subsection{Patient-Specific Image Registration}

To improve the realism of eye surgery simulation, it is high important to integrate patient-specific eye disease information into the simulator. Ophthalmologic images are embedded inside the 3D parametric model using the atlas-based image alignment algorithm [11-12]. A uniform polar coordinate system of the eyeball is used in the process. A texture mapping technique is applied to match the ophthalmologic images and the eyeball structure. Alignment of the ophthalmologic image with the eyeball under the guidance of eye atlas is thus turned into an optimization task. 
With patient specific ophthalmologic images embedded into the $3 \mathrm{D}$ eyeball model, visualization of abnormal eyeball structure can be performed. Figure 4 illustrates the visual field of a glaucoma eye disease.
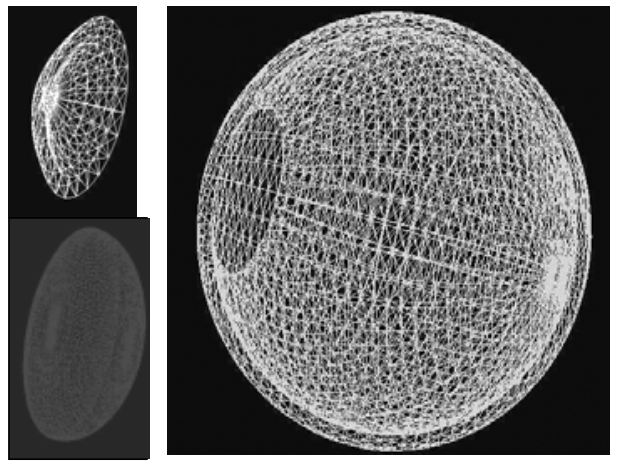

Figure 3. Parametric representation of eyeball model

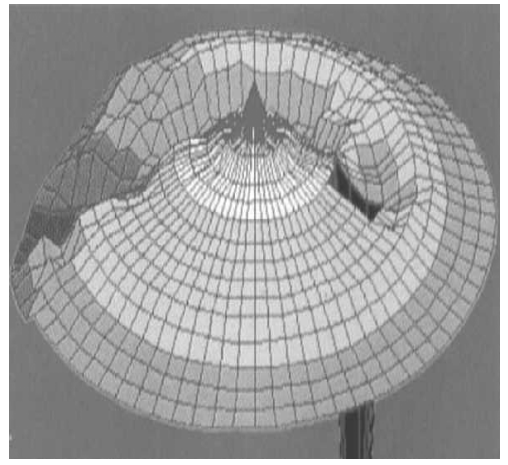

Figure 4. Visualization of eye abnormal

\section{FEM-Based Physical Modeling for Eye Surgery}

We are developing a computer-based simulator for eye surgery to help medical students and junior ophthalmologists impeove their surgical skills. We have built a physical based model with finite element method to simulate the real movement of bio-structures in response to the manipulation of the instruments during the surgical procedure. The visco-elasticity biomechanics is adopted to describe the deformation of the bio-structures [13-15]. In the 3D ocular model, we have considered the following structures: (1) Cornea, (2) Lens, (3) Sclera, (4) Choroids, and Iris, (5) Retina, (6) Muscles.

All the structures are parametrically modeled with reference to the ophthalmologic knowledge base. Emphasis, however, will be placed upon the cornea, lens, and choroids since these are the primary structures involved in common practices. In the following sections, we describe the bio-mechanical modeling of instruments and eye bio-structures.

\subsection{Movement of Instrument}

It is noted that the elasticity of all the instruments in practices are much harder then all the bio-structures involved in the operation. It is assumed that a rigid structure with six-degree of freedom is used to represent the instruments. The movements of the instruments are then the sum of rigid body displacements as

$$
\mathfrak{R}=\mathfrak{R}\left(R_{x}, R_{y}, R_{z}, R_{\theta}, R_{\rho}, R_{\omega}\right)
$$


where $R_{x}, R_{y}$ and $R_{z}$ are the coordinates of the end of the instruments at the global space. $R_{\theta}, R_{\rho}$ and $R_{\omega}$ represent the two rotations of the tip of the instruments with respect to the axis of the global system and a rotation with regard to its logistical axis.

\subsection{Governing Equation of Deformation of Biostructures}

From studies, $90 \%$ of corneal thickness is formed by the stroma. We assumed that this controls the majority of the biomechanical behavior of the cornea and mainly consists of water $(78 \%)$ and layered protein fibres $(16 \%)$, which provides strength, elasticity and form. Ideally, it is assumed as the visco-elasticity material in the deformation analysis.

A visco-elastic material is one which exhibits properties of both viscous fluids and elastic solids. Many materials exhibit properties of elastic and viscous behavior dependent on stress. Examples include metals and Bingham plastics. However, viscoelasticity describes a material which exhibits a combination of viscous and elastic behavior simultaneously. Visco-elastic materials can appear to be either solid or liquid, depending on whether the dominant property is elastic or viscous. Viscoelasticity is common among macromolecular materials and is often the result of molecular entaglement between macromolecules. Visco-elastic materials are characterized by two particular behaviors: Creep and Stress Relaxation.

When a sudden load is applied, there is an initial rapid extension but instead of having a fixed extension as with an elastic solid, it continues to extend with time. When the load is removed, there is an initial rapid contraction that quickly slows down. Conversely, the stress required to maintain constant strain decreases with time. This is known as stress relaxation. And the deformation problem we encountered here is a multi-structures stress relaxation problem.

\subsection{FEM Formulation}

We consider the FEM analysis of this multi-structures stress relaxation problem. The materials here is a time-dependent "flow" occurrence due to the intervention of the instruments. The given equation can be given as, using Hamilton's principle,

$$
F\left(x_{i}, t\right)=\int_{t_{0}}^{t_{1}} L d t=\int_{t_{0}}^{t_{1}}(\Pi-\Lambda) d t
$$

Where the Lagrangian function $\mathrm{L}$ is defined in terms of the potential energy $(\Pi)$ and the Kinetic energy $(\Lambda)$.

The calculation of $\Pi$ can be made:

$$
\Pi=\frac{1}{2} d^{T} \int_{0}^{v} B^{T} D B d v d
$$

where

$$
d=\left[d_{1 x} d_{1 y} d_{1 z}, d_{1 x} d_{1 y} d_{1 z}, \cdots, d_{N x} d_{N y} d_{N z}\right]^{T}
$$


$d_{i x}, d_{i y}, d_{i z}$ are the displacements at $\mathrm{x}, \mathrm{y}, \mathrm{z}$ directions at $i^{\text {th }}$ FEM node. $\mathrm{B}$ is the standard strain matrix and $\mathrm{D}$ is the material matrix. $\Pi$ can be further written as

$$
\Pi=\frac{1}{2} d^{T} \mathrm{~K} d
$$

where

$$
\mathrm{K}=\int_{0}^{v} B^{T} D B d v
$$

$\mathrm{K}$ is the stiffness matrix of the bio-structures.

The kinetic energy $\Lambda$ can be given as

$$
\Lambda=\frac{1}{2} \int_{0}^{v} \rho\left(\frac{\partial d}{\partial t}\right)^{2} d v
$$

$\rho$ is the mass density of the individual material.

Our problem here is a First-order problem as

$$
\mathrm{K} \dot{d}+\mathrm{K} d=Q
$$

where

$$
\dot{d}=\frac{\partial d}{\partial t}
$$

and these derivatives can be replaced by finite difference approximations. In the Crank-Nicokon scheme, a time-stepping technique,

$$
\begin{aligned}
& d=\frac{d_{n+1}+d_{n}}{2} \\
& \dot{d}=\frac{d_{n+1}-d_{n}}{\delta}
\end{aligned}
$$

where $\delta$ is the finite step length.

Hence the recurrence relation is obtained as

$$
\left(\mathrm{K}+\frac{2}{\delta} \mathrm{K}_{d}\right) d_{n+1}=Q_{n+\frac{1}{2}}+\left(\frac{2}{\delta} \mathrm{K}_{d}-\mathrm{K}\right) d_{n}
$$

It is implemented, for consistency, equation (10) should be used for Q. And using backward difference for (11) giving

$$
\left(\mathrm{K}+\frac{1}{\delta} \mathrm{K}_{d}\right) d_{n+1}=Q_{n+1}+\frac{1}{\delta} \mathrm{K}_{d} d_{n}
$$

In this case, the displacement increments $\mathrm{d}$ are calculated using the equilibrium iteration.

\section{Virtual Reality Supported Eye Surgical Simulation}

We developed a computer-based simulator for eye surgery with the parametric eyeball model to help medical students and ophthalmologists improving their surgical skills. 
Figure 5(a) is an image of the rendered eyeball model. Figures 5(b) is a snapshot of the graphical user interface. It shows the interaction between the device and the cornea surface of the eyeball modeled. User can feel the force feedback on the device via the force feedback device (PHANToM) in our simulation environment shown in Figure 6.

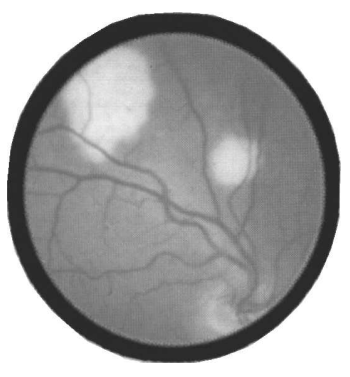

(a)

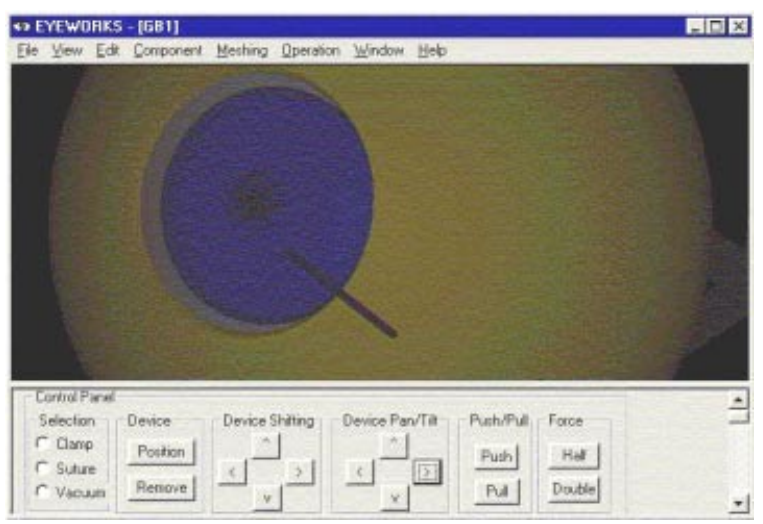

(b)

Figure 5. (a) Eyeball model (b) Snapshot of graphical user interface of simulator

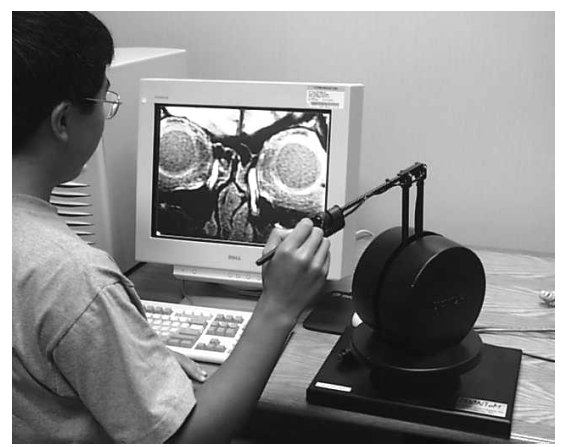

Figure 6. Eye surgical simulation environment

The simulation system provides direct interaction with patient-specific model and volumetric data set while delivering haptic feedback to the user. A real-time volume rendering technique based on ray casting, trilinear interpolation, and per-sample gradient estimation algorithms was employed to visualize the patient-specific volume data. Our physical-based eyeball model and the associated FEM analysis provide the realistic haptic rendering. User feels the force through the PHANToM. With the guidance of see-able and magnified internal eyeball 3D representations of the patientspecific data, a trainee can easily interact with the abnormal eye structures such as slightly cutting some cornea with a virtual scalpel. In Figure 6, a user is manipulating the PHANToM to interact with a patient's eyeball volume MRI data sets under the assistance of a variety of virtual tools. 


\section{Conclusion}

To meet the needs of training for ophthalmological professionals, a prototype for eye surgery simulation is described using the physical-based parametric eyeball model. It enables simplified eye surgery simulation in a real-time mode and with an easy-to-use feature. Future works includes building up more accurate and realistic eye models. More devices and eye surgical operations will be developed. In order to allow users to realistically operate the simulated tools, appropriate hand-eye co-ordination will be enhanced through continue application of virtual reality technologies.

\section{Acknowledgements}

Support of this research development by National Science and Technology Board of Singapore is gratefully acknowledged. The project is partially sponsored by the Nanyang Technological University (SDS 15/2000).

\section{Reference}

[1] Mann, I. C., "Culture, Race, Climate and Eye Disease : An Introduction to The Study of Geographical Ophthalmology", Springfield, Ill. : Thomas, 1966.

[2] Lim A. S. M., "Ophthalmology", Singapore Society of Ophthalmology, Singapore, 1982.

[3] Hanna, K. D., Jouve, F. E., Waring III, G. O., and Ciarlet, P. G., Computer simulation of arcuate and radial incisions involving the corneoscleral limbus, Eye, 1989, No. 3, pp. 227 239.

[4] Hanna, K. D., Jouve, F. E., Waring III, G. O., and Ciarlet, P. G., Computer simulation of arcuate and keratotomy for astigmatism, Refractive \& Corneal Surgery, Vol. 8, March/April 1989, pp. 152-163.

[5] Sagar, M. A., Bullivant, D., Mallinson, G. D., Hunter, P. J., and Hunter, I. W., A virtual environment and model of the eye for surgical simulation, Proceedings of SIGGRAPH Annual Computer Graphics Conference 1994, Orlando, Florida, July 24-29, pp. 205-211.

[6] Hunter, I. W., Doukogiou, D., Lafontaine, S. R., Charette, P. G., Jones, L. A., Sagar, M. A., Mallinson, G. D., and Hunter, P. J., A teleoperated microsurgical robot and associated virtual environment for eye surgery, Presence, Vol. 2, No. 4, MIT, Fall 1993, pp. 265-280.

[7] Davson, H., "The Eye", Academic Press, Cincinnati, New York, USA, 1984.

[8] Spencer W. H., "Ophthalmic Pathology", W.B. Saunders Co., Philadelphia, 1996.

[9] Duane, T. D. and Jaeger E. A., "Biomedical Foundations of Ophthalmology", Harper \& Row, Philadelphia, PA, 1986.

[10] Freberg, T. R. and Lace, J. W., A comparison of the elastic properties of human choroids and sclera, Experiment Eye Research, Vol. 47, 1988, pp. 429-436.

[11] Richard, K. and Parrish II, Bascom Palmer Eye Institute's Atlas of Ophthalmology, Philadelphia, PA, Current Medicine, 1999.

[12] Perkins, E. S., An Atlas of Diseases of the Eye, Churchill Livingstone, Edinburgh, 1987.

[13] van Alphen, G. W. H. M., and Graebel, W. P., Elasticity of tissues involved in accommodation, Vision Research, Vol. 31, No. 7/8, 1991, pp. 1417-1438.

[14] Gallagher R. H., et al, "Finite Elements In Biomechanics", Wiley, New York, USA, 1982.

[15] Pinsky, P. M., and Datye, D. V., A microstructureally-based finite element model of the incised human cornea, Bio-mechanics, Vol. 24, No. 10, 1991, pp. 907-922. 